J Miner Stoffwechs Muskuloskelet Erkrank $2017 \cdot 24: 101$

https://doi.org/10.1007/s41970-017-0015-0

๑) Springer-Verlag GmbH Austria 2017

CrossMark

\title{
Christian Dejaco
}

Dienst für Rheumatologie, Südtiroler Sanitätsbetrieb, Krankenhaus Bruneck, Bruneck, Italien

\section{Editorial}

Liebe Kolleginnen und Kollegen, liebe Freunde der Rheumatologie!

Wieder können wir stolz sein auf die Forschungsleistungen der österreichischen Rheumatologie. Es wurden insgesamt 65 Abstracts eingereicht, davon 13 in der Kategorie „Fall des Jahres“, bei dem die interessantesten rheumatologischen Fallberichte des Jahres 2017 vorgestellt werden. Die Forschungsarbeiten stammen, wie auch in den letzten Jahren, aus den verschiedensten Bereichen der Rheumatologie wie der Pathophysiologie, klinischen Studien, der Rehabilitation oder der Kinderrheumatologie und wurden von Kolleginnen und Kollegen aus allen Landesteilen verfasst. Diese Vielfalt widerspiegelt einerseits, wie „bunt“ die Rheumatologie geworden ist, andererseits wie viele engagierte Kolleginnen und Kollegen daran arbeiten, die Betreuung unserer Patienten zu verbessern.

Ich möchte an dieser Stelle auch wieder die Gelegenheit nutzen, im Namen der wissenschaftlichen Sektion und des gesamten Vorstandes der Österreichischen Gesellschaft für Rheumatologie und Rehabilitation (ÖGR) allen zu danken, die zum Gelingen der Jahrestagung 2017 beigetragen haben. Neben dem Organisationsteam sind es vor allem Sie, die Sie als Referenten, Moderatoren und/ oder Autoren von Abstracts die Jahrestagung spannend und lehrreich gestalten. Auch dem Verlag, der die Jahrestagung und unsere Gesellschaft unterstützt, gilt mein besonderer Dank!

Ich wünsche Ihnen eine interessante und abwechslungsreiche ÖGR-Jahrestagung 2017!

Mit kollegialen Grüßen, Christian Dejaco

\section{Korrespondenzadresse

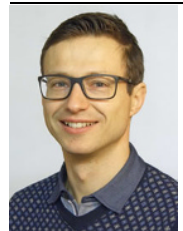 \\ Assoz. Prof. PD Dr. med. univ. C. Dejaco, Ph.D., MBA \\ Dienst für Rheumatologie, Südtiroler Sanitätsbetrieb, Krankenhaus Bruneck Spitalstraße 11, 39031 Brun- eck, Italien christian.dejaco@sabes.it}

Interessenkonflikt. C. Dejaco gibt an, dass kein Interessenkonflikt besteht. 